\title{
Sawing Through The Neck - A Case of Serrated Fish Bone Migrating to Prevertebral Space
}

\author{
Ngui LXa , Shashi G. ${ }^{b}$, Muhammad Nasri AB. ${ }^{b}$
}

aDepartment of ORL-HNS, Sibu Hospital

bepartment of ORL-HNS, Sibu Hospital

\section{ABSTRACT}

Fish bone is the commonest pharyngeal foreign body, however migrating fish bone is a rare occurrence. We report a case of a 54-year-old male who had history of fish bone ingestion over a week and presented with odynophagia and worsening neck pain. Rigid esophagoscopy revealed tip of a fish bone which was embedded in granulation tissue. The fish bone migrated further with manipulation. Aided with computed tomography scan findings, the serrated fish bone was finally removed via transcervical approach without any complication. In conclusion, high index of suspicion and prompt removal of migrating fish bone with the aid of computed tomography imaging is necessary to avoid fatal complications.

KEYWORDS: Fish bone, Foreign body, Migration, Prevertebral space

\section{INTRODUCTION}

Accidental fish bone ingestion is a common occurrence. According to Chai CK et al, fish bone is the commonest pharyngeal foreign body in Sarawak General Hospital which constituted for $92.8 \%$ of pharyngeal foreign body cases seen. Fish bone also contributed to a total of $29.8 \%$ of oesophageal foreign body. ${ }^{1}$ We would like to report our experience in managing a case of serrated fish bone migrating to prevertebral space which was successfully removed via external approach without any complication.

\section{CASE REPORT}

A 54-year-old gentleman presented to Department of Otorhinolaryngology, Head and Neck Surgery (ORL-HNS) of Sibu Hospital for odynophagia and worsening neck pain over a week. He gave a history of fish bone ingestion of one week duration with odynophagia, but was still able to tolerate orally. Over the next few days, the patient developed pain

\section{Corresponding Author}

Dr. Ngui Ling Xiu

Department of Otorhinolaryngology,

Head and Neck Surgery, Sibu Hospital

KM 5 1/2, Jalan Ulu Oya,

96000 Sibu, Sarawak, Malaysia

Tel no : +6016-9061979

E-mail : nguilingxiu@hotmail.com over anterior part of the neck and low grade fever which was when he sought further treatment. Upon examination, patient was afebrile with tenderness over anterior part of the neck. Flexible laryngoscopy done did not reveal any signs of foreign body. There was no obvious foreign body seen on lateral neck radiography.

Intravenous Augmentin was commenced immediately. We proceeded with rigid esophagoscopy which revealed firm granulation tissue over the right posterolateral wall of esophagus, about $17 \mathrm{~cm}$ from the upper incisor. Tip of a fish bone was seen within the granulation tissue and we attempted to remove it with grasping forceps. However, the fish bone was lost during manipulation. The procedure was abandoned and Ryle's tube was inserted for feeding.

A computed tomography (CT) scan was arranged for him. The scan revealed a $3.0 \mathrm{~cm} \times 0.3 \mathrm{~cm}$ hyperdense, linear foreign body in the right prevertebral soft tissue at the level of C7/T1 vertebral body, projecting anterolaterally and inferiorly. (Fig.1) Its medial end laid $0.9 \mathrm{~cm}$ anterior to the $\mathrm{C} 7 / \mathrm{T} 1$ intervertebral disc. (Fig.2) Its lateral end laid posterior to the right common carotid artery and was the most superficial aspect, measuring $2.3 \mathrm{~cm}$ from the skin. No active contrast extravasation was seen from the adjacent vessels. No rim enhancing collection was seen in the prevertebral soft tissue to suggest abscess. 


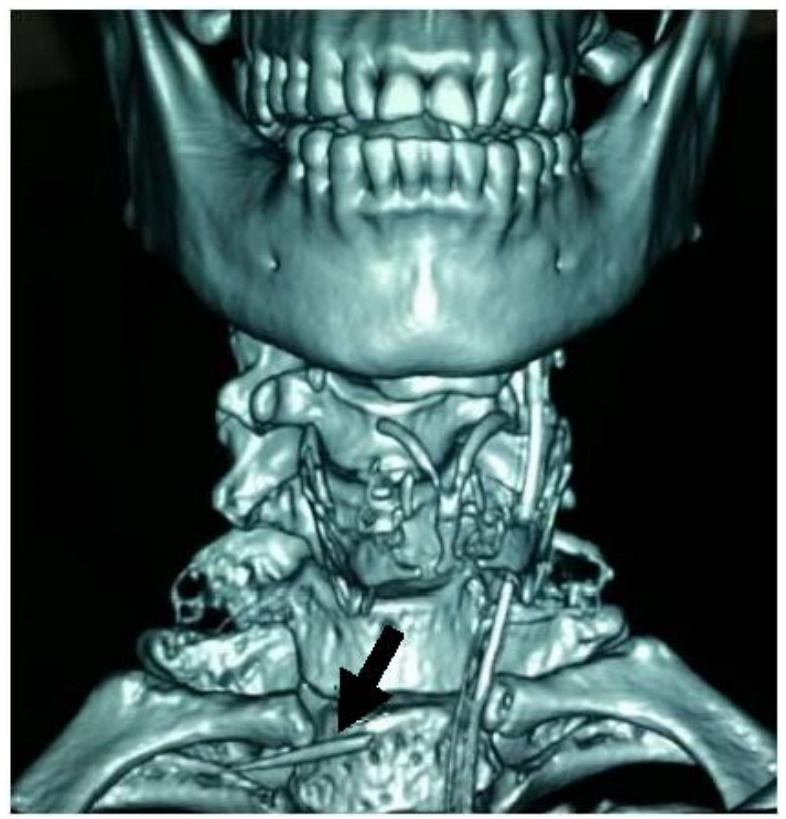

Figure $13 \mathrm{D}$-reconstruction of a computed tomography of the neck shows a $3.0 \mathrm{~cm} \times 0.3 \mathrm{~cm}$ hyperdense, linear foreign body in the right prevertebral soft tissue at the level of C7/T1 vertebral body, projecting anterolaterally and inferiorly. (black arrow)

He was subjected to neck exploration on the same day via transcervical approach. Along the track to locate the fish bone; the right thyroid gland was retracted medially while the right inferior thyroid artery was ligated and the right common carotid artery was retracted laterally. A serrated fish bone was found deeply embedded in the preverterbral fascia about $0.5 \mathrm{~cm}$ to the lateral border of esophagus. The fish bone was successfully removed in total. (Fig. 3) He was discharged with a nasogatric tube which was eventually removed one week post neck exploration and oral feeding was commenced.

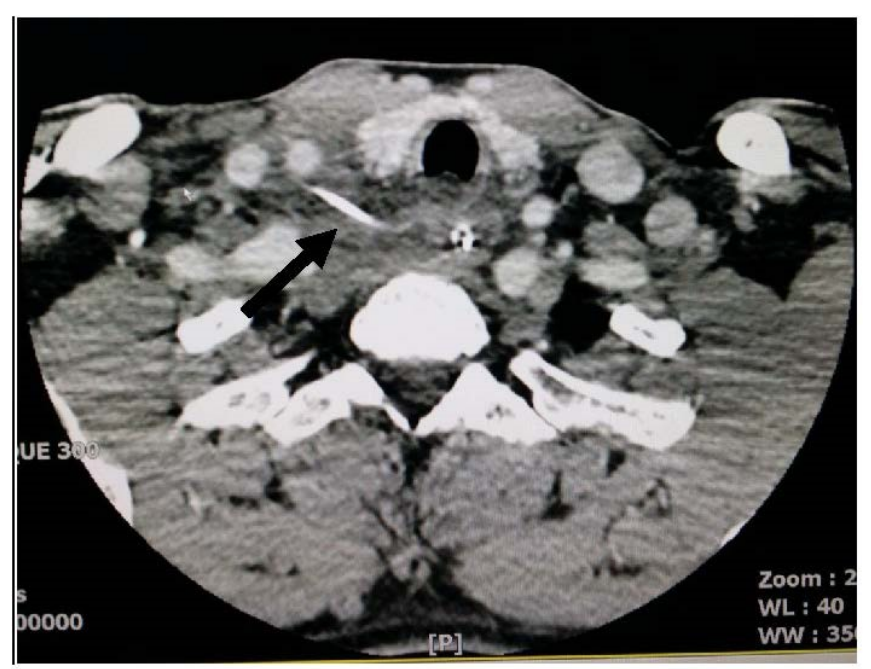

Figure 2 Computed tomography of the neck shows the position of the fish bone (black arrow), with its medial end anterior to the $\mathrm{C} 7 / \mathrm{T} 1$ intervertebral disc and lateral end posterior to the right common carotid artery.

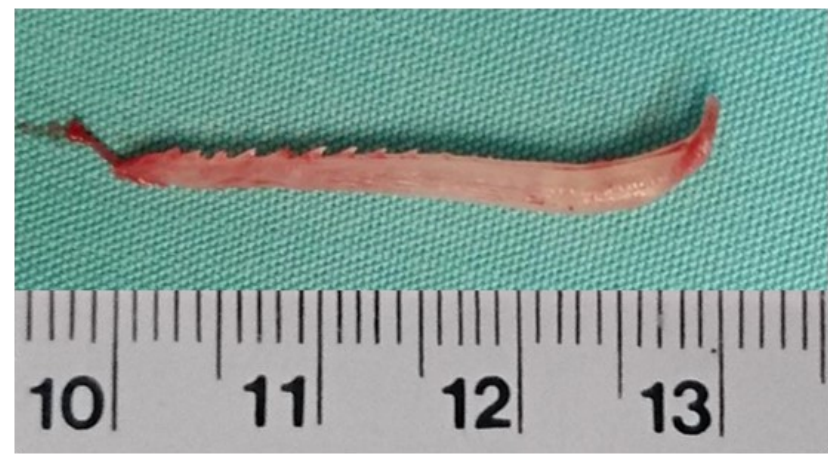

Figure 3 The serrated fish bone after removal, measuring $3.0 \mathrm{~cm}$, showing unidirectional saw-tooth on one side.

\section{DISCUSSION}

Patients who have ingested fish bone typically present acutely in emergency setting. Over $60 \%$ of patients with oesophageal foreign body ingestion are symptomatic within the first 24 hours, while $80 \%$ present within the first week. ${ }^{2}$ Most of the fish bone will be lodged over the tonsils, base of tongue and vallecula, which can be visualized directly or with the aid of laryngoscope and can be removed instantly. However, sharp and linear fish bones have a tendency to migrate out of the digestive tract; commonly at cervical oesophagus, followed by upper thoracic oesophagus and rarely migrating out of intestines. ${ }^{3,4}$

Oesophageal movements during swallowing, neck muscles contraction and manipulations during endoscopy facilitate the migration of fish bones into the neck. Fish bones have been reported to migrate to thyroid gland, piercing through common carotid artery and internal jugular vein, and through cervical spine to posterior subcutaneous tissue. ${ }^{5,6}$ Fish bone migration can present with complications of deep neck abscess, hematoma due to vascular injury and rarely mediastinitis. ${ }^{7}$

Oral examination and laryngoscopy are usually nondiagnostic for migratory fish bone, as was the case with our patient. Plain radiography of lateral neck was performed was also not helpful. Although cheap and easily available, the sensitivity of lateral neck radiographs in the detection of foreign body is reported to be as low as $25.3 \% .^{8}$ Location of impaction, orientation, and density of the foreign body affects the visualization of foreign body on the lateral neck radiograph. ${ }^{9}$

Alternatively, the sensitivity of multidirectional computed tomography (MDCT) to detect an 
impacted foreign body ranged from 90 to $100 \%$ with a specificity of 93.7 to $100 \%$, making it significantly superior to plain radiography in detecting foreign body. ${ }^{10}$ Furthermore, CT scan elucidates objectively the size, type, accurate location and orientation of the foreign body with the surrounding vital structures; making it easier for surgeons to determine the best surgical approach to remove the foreign body.

In our case, the presence of foreign body was confirmed when the tip of the fish bone seen within the granulation tissue during the rigid endoscopy examination. However, even without visible foreign body, the presence of mucosal tear or granulation tissue should raise the suspicion for migratory foreign body. In our case, the migrated fish bone which was partly seen intraluminally failed to be removed through rigid endoscopy, and manipulation caused the fish bone to migrate further. This should be attributed to the nature of the fish bone which was serrated. Serrated fish bone with unidirectional saw-tooth on its sides allows only unidirectional movement, promoting deeper penetration and prevents it from being withdrawn intraluminally.

\section{CONCLUSION}

Migrating fish bone is a rare occurrence as compared to high incidence of fish bone ingestion. Partially migrated fish bone may be extracted via intraluminal approach although in rare conditions where the fish bone is serrated; external approach and neck exploration are required. Migrating fish bone in neck may cause fatal complications if left unattended even though spontaneous extrusion of migrating fish bone has been reported. Therefore, high index of suspicion and prompt removal of migrating fish bone with the assistance of CT scan is crucial.

\section{Conflicts of interest:}

We have no conflict of interest to declare.

\section{Consent:}

Informed consent has been obtained from the patient for the purpose of publication.

\section{REFERENCES}

1. Chai CK, Tang IP, Tan TY, Jong DE. A review of ear, nose and throat foreign bodies in Sarawak General Hospital. A five year experience. Med J
Malaysia 2012; 67: 17-20.

2. Macpherson RI, Hill JG, Othersen HB, Tagge EP, Smith CD. Esophageal foreign bodies in children: diagnosis, treatment, and complications. AJR Am J Roentgenol 1996; 166: 919-924.

3. Loh KS, Tan LK, Smith JD, Dong F. Complications of foreign bodies in the esophagus. Otolaryngol Head Neck Surg 2000; 123: 613-616.

4. Beecher SM, O'Leary DP, McLaughlin R. Diagnostic dilemmas due to fish bone ingestion: Case report \& literature review. Inter J of Surg Case Reports 2015; 13: 112-115.

5. Tang IP, Shashinder S, Shoba N, Rahmat O, Shivalingam S, Gopala KG, et al. Migrating foreign body into the common carotid artery and internal jugular vein - A rare case. Auris Nasus Larynx 2009; 36: 380-382.

6. Bilish DP, Rajan GP, Farooque F. Fish bone migration through the cervical spine. Otolaryngol Head Neck Surg 2008; 138: 407-408.

7. Jha SK, Kumar SP, Somu L, Ravikumar A. Missing fish bone: case report and literature review. Am J Otolaryngol 2012; 33: 623-626.

8. Evans RM, Ahuja A, Rhys Williams S, Van Hasselt CA. The lateral neck radiograph in suspected impacted FBs-does it have a role? Clin Radiol 1992; 46: 121-123.

9. Lue AJ, Fang WD, Manolidis S. Use of plain radiography and computed tomography to identify FB foreign bodies. Otolaryngol Head Neck Surg 2000; 123: 435-438.

10. Liew CJY, Poh ACC, Tan TY. Finding nemo imaging findings, pitfalls, and complications of ingested fish bones in alimentary canal. Emerg Radiol 2013; 20: 311-322. 